\title{
Solution of Mixed Problem Including a First Order Three Dimensional P.D.E with Nonlocal and Global Boundary Conditions
}

\author{
N.A. Aliev , O.H. Asadova, A.M. Aliev* \\ Baku State University, 23, Z. Khalilov str., AZ1148, Baku, Azerbaijan \\ Corresponding Author: aahmad07@rambler.ru
}

Copyright @ 2013 Horizon Research Publishing All rights reserved.

\begin{abstract}
In this paper solution of mixed complex boundary value problem of first order is considered. The basic term in the problem with respect to space variables, has Cauchy-Riemann operator. We first use Laplace transformation to introduce spectral problem. Then we investigate for corresponding Fredholm's type. The spectral problem here is different from classical boundary value problems. Here boundary conditions are nonlocal and global and in general linear.At the end we find asymptotic expansionfor the solution of spectral problemwhich depends on unknown complex parameter. With the help of this asymptotic expansion we prove existence and uniqueness of mixed problem.
\end{abstract}

Keywords Mixed Problem, Nonlocal And Global Boundary Conditions, Singularity, Complex Valued Boundary Value Conditions, Regularization, Fredholm's Type, Asymptotic Expansion

\section{Introduction}

Let time $t \in(0, \infty)$, space variables $x=\left(x_{1}, x_{2}\right) \in I R^{2}$ and $D \subset I R^{2}$ be a bounded connected region. $\Gamma=\bar{D} \backslash D$ is considered as a boundary of $D$ where every vertical line to $x_{1}$ axis can intersect the boundary at most at two points. Here we assume that $x_{1}$ axis divides $\Gamma$ in two curves $\Gamma_{1}, \Gamma_{2}$ respectively. Equations for $\Gamma_{1}$ and $\Gamma_{2}$ are given by

$$
x_{1} \in\left[a_{1}, b_{1}\right], x_{2}=\gamma_{k}\left(x_{1}\right) ; k=1,2 .
$$

Consider the following complex, linear partial differential equation

$$
\begin{gathered}
\frac{\partial u(x, t)}{\partial t}=\frac{\partial u(x, t)}{\partial x_{2}}+i \frac{\partial u(x, t)}{\partial x_{1}}+a(x) u(x, t)+f(x, t), \\
x \in D \subset I R^{2}, t>0,
\end{gathered}
$$

with boundary condition

$$
\begin{gathered}
\alpha_{1}\left(x_{1}\right) u\left(x_{1}, \gamma_{1}\left(x_{1}\right), t\right)+\alpha_{2}\left(x_{1}\right) u\left(x_{1}, \gamma_{2}\left(x_{1}\right), t\right)+ \\
\int_{a_{1}}^{b_{1}}\left[K_{1}\left(x_{1}, \xi\right) u\left(\xi_{1}, \gamma_{1}\left(\xi_{1}\right), t\right)+K_{2}\left(x_{1}, \xi_{1}\right) u\left(\xi_{1}, \gamma_{2}\left(\xi_{1}\right), t\right)\right] d \xi_{1}+ \\
\int_{D} K\left(x_{1}, \xi\right) u(\xi, t) d \xi=\alpha\left(x_{1}, t\right)
\end{gathered}
$$




$$
x_{1} \in\left[a_{1}, b_{1}\right] \subset I R, t \geq 0
$$

and initial value

$$
u(x, 0)=\varphi(x), x \in \bar{D}
$$

where $a(x), f(x, t), \alpha_{j}\left(x_{1}\right), j=1,2, \phi(x)$ and $\alpha\left(x_{1}, t\right)$ are given continuous functions. $K_{j}\left(x_{1}, \xi_{1}\right)$ for $j=1,2$ and $K\left(x_{1}, \xi\right)$ are given continuous kernels or they contain weak singularity. $\Gamma$ the boundary of $D$ is Lyapunov curve or piecewise Lyapunov. Let $x_{2}=\gamma_{k}\left(x_{1}\right), k=1,2$ as a curve $\Gamma_{k}$ be differentiable and its differential is of Holder class.

Mixed partial differential equations are basically considered for parabolic (Heat equation) and hyperbolic (wave equation) kinds of problems ([4], [5], [6]). In these cases number of boundary conditions (for local conditions) is the half of highest order of derivative of unknown function with respect to space variables, (for even orders), ([4], [5], [6]).

The problem of investigation in this paper is basically aelliptic equation, i.e. Cauchy-Riemann equation. Note that in classical mathematical physics problems, the simplest canonical elliptic equation is Laplace equation, which is of second order. Here our problem is of first order with mixed boundary conditions.

Here, if the boundary condition is Dirichlet's (with any unknown equation in all over the boundary $\Gamma$ ) then the problem has no solution, i.e. the problem is not well posed. In this problem boundary condition is nonlocal and the number of boundary conditions is equal to highest order of derivative with respect to space variables ([7], [8], [9]). This phenomenon is similar to ordinary differential equations.

\section{Spectral Problem}

Applying Laplace transformation ([4], [9]) to mixed problem (1-3) gives:

$$
\begin{gathered}
\int_{0}^{\infty} e^{-\lambda t} \frac{\partial u(x, t)}{\partial t} d t=\int_{0}^{\infty} e^{-\lambda t} \frac{\partial u(x, t)}{\partial x_{2}} d t+i \int_{0}^{\infty} e^{-\lambda t} \frac{\partial u(x, t)}{\partial x_{1}} d t+ \\
+\int_{0}^{\infty} e^{-\lambda t} a(x) u(x, t) d t+\int_{0}^{\infty} e^{-\lambda t} f(x, t) d t
\end{gathered}
$$

where $\lambda=c+i \tau$ is a complex parameter, $c>0$ and $\tau \in I R$. Now, considering (3) and integrating by parts from left hand side of above equation and by accepting

$$
\begin{aligned}
& \int_{0}^{\infty} e^{-\lambda t} u(x, t) d t=\tilde{u}(x, \lambda), x \in D \\
& \int_{0}^{\infty} e^{-\lambda t} f(x, t) d t=\widetilde{f}(x, \lambda), x \in D
\end{aligned}
$$

we can write

$$
\ell \tilde{u} \equiv \frac{\partial \tilde{u}(x, \lambda)}{\partial x_{2}}+i \frac{\partial \tilde{u}(x, \lambda)}{\partial x_{1}}-\lambda \tilde{u}(x, \lambda)=F(x, \lambda), x \in D
$$

where

$$
F(x, \lambda)=-\tilde{f}(x, \lambda)-\varphi(x)-a(x) \tilde{u}(x, \lambda), x \in D
$$

Similarly we may find from boundary condition (2) that

$$
\alpha_{1}\left(x_{1}\right) \tilde{u}\left(x_{1}, \gamma_{1}\left(x_{1}\right), \lambda\right)+\alpha_{2}\left(x_{1}\right) \tilde{u}\left(x_{1}, \gamma_{2}\left(x_{1}\right), \lambda\right)+
$$




$$
\begin{array}{r}
\int_{a_{1}}^{b_{1}}\left[K_{1}\left(x_{1}, \xi_{1}\right) \tilde{u}\left(\xi_{1}, \gamma_{1}\left(\xi_{1}\right), \lambda\right)+K_{2}\left(x_{1}, \xi_{1}\right) \tilde{u}\left(\xi_{1}, \gamma_{2}\left(\xi_{1}\right), \lambda\right)\right] d \xi_{1}+ \\
\int_{D} K\left(x_{1}, \xi\right) \tilde{u}(\xi, \lambda) d \xi \equiv \tilde{\alpha}\left(x_{1}, \lambda\right), \quad x_{1} \in\left[a_{1}, b_{1}\right],
\end{array}
$$

where

$$
\widetilde{\alpha}\left(x_{1}, \lambda\right)=\int_{0}^{\infty} e^{-\lambda t} \alpha\left(x_{1}, t\right)
$$

The problem found from (6) and (8) is called spectral problem dependent on mixed problem (1-3). Since in this problem we do not have any derivative with respect to $\lambda$ we call $\lambda$ as a spectral parameter.

Remark 1: In the spectral problem (6) and (8) the continuity of kernels $K\left(x_{1}, \xi\right)$ and $K_{j}\left(x_{1}, \xi_{1}\right), j=1,2$, is of great importance. If they are not continuous, they are dependent to linear combination of Dirac delta-functions. Then it is possible to determine minimal and maximal operators for (6) in region $D$ [9]. In this case we may find arbitrary operator between minimal and maximal operator from (6) and (8). In the other words, arbitrary operator may be given with help of boundary condition (8) by squeezing down the domain of maximal operator. This is in contradiction with whatever we assume in functional analysis when we are working with theory of operators dependent to differential equations, i.e. we assume that it is not possible to find ordinary operator between maximal and minimal operators ([9], [10], [16]).

\section{Finding Necessary Conditions}

It is obvious that equation for adjoint problem of spectral problem (6) and (8) is in the form

$$
\ell^{*} V \equiv-\frac{\partial V(x, \lambda)}{\partial x_{2}}+i \frac{\partial V(x, \lambda)}{\partial x_{1}}-\bar{\lambda} V(x, \lambda)=G(x, \lambda), x \in D
$$

where $G$ is an arbitrary function ([3], [4]).

In order to find fundamental solution for equation (10), we use Fourier transformation ([3], [11]), then we have

$$
V(x-\xi, \lambda)=\frac{1}{2 \pi i} \cdot \frac{e^{-i c\left(x_{1}-\xi_{1}\right)+i \tau\left(x_{2}-\xi_{2}\right)}}{x_{1}-\xi_{1}+i\left(x_{2}-\xi_{2}\right)}
$$

where

$$
-\frac{\partial V(x-\xi, \lambda)}{\partial x_{2}}+i \frac{\partial V(x-\xi, \lambda)}{\partial x_{1}}-\bar{\lambda} V(x-\xi, \lambda)=\delta(x-\xi)
$$

while $\delta(x-\xi)$ is a two-dimensional Dirac delta-function.

Theorem 1: Let region $D$ be a connected bounded area in $I R^{2}$, and its boundary $\Gamma$ be of Lyapunov curve type (or it is piecewise Lyapunov curve). Assume every vertical line to $x_{1}$ axis, intersect the boundary $\Gamma$ in at most two points.

Moreover, if $\Gamma(x, \lambda)$ is a continuous function, then every solution to (6) in $\bar{D}$ is satisfying (13).

Proof: Following the technique in [7] and [8] we try to find arbitrary solution for (6) in a given region $D$. We are looking for those necessary conditions which the solution itself satisfy in them. Multiplying both sides of (6) by $\bar{V}(x-\xi, \lambda)$ and integrating over region $D$ we may write

$$
\begin{aligned}
& \int_{D} \frac{\partial \tilde{u}(x, \lambda)}{\partial x_{2}} \bar{V}(x-\xi, \lambda) d x+i \int_{D} \frac{\partial \tilde{u}(x, \lambda)}{\partial x_{1}} \bar{V}(x-\xi, \lambda) d x- \\
& \lambda \int_{D} \tilde{u}(x, \lambda) \bar{V}(x-\xi, \lambda) d x=\int_{D} F(x, \lambda) \bar{V}(x-\xi, \lambda) d x .
\end{aligned}
$$


Applying Ostrogradsky-Gauss formula to the first two terms of above equation or integrating by parts ([3], [4]) gives

$$
\begin{gathered}
\int_{\Gamma} \tilde{u}(x, \lambda) \bar{V}(x-\xi, \lambda)\left[\cos \left(n, x_{2}\right)+i \cos \left(n, x_{1}\right)\right] d x+ \\
\int_{D} \tilde{u}(x, \lambda)\left[-\frac{\overline{\partial V(x-\xi, \lambda)}}{\partial x_{2}}+i \frac{\partial V(x-\xi, \lambda)}{\partial x_{1}}-\bar{\lambda}(x-\xi, \lambda)\right] d x= \\
\int_{D} F(x, \lambda) \bar{V}(x-\xi, \lambda) d x
\end{gathered}
$$

where $n$ is the outer normal vector at point $x$ on $\Gamma$. Now using (12) and properties of Dirac delta function ([3], [7]) we will find

$$
\begin{gathered}
\int_{D} F(x, \lambda) \bar{V}(x-\xi, \lambda) d x-\int_{\Gamma} \tilde{u}(x, \lambda) \bar{V}(x-\xi, \lambda)\left[\cos \left(n, x_{2}\right)+i \cos \left(n, x_{1}\right)\right] d x= \\
\left\{\begin{array}{l}
\tilde{u}(\xi, \lambda) ; \quad \xi \in D \\
\frac{1}{2} \tilde{u}(\xi, \lambda) ; \xi \in \Gamma .
\end{array}\right.
\end{gathered}
$$

Thus the solution is received in a form dictated by the second Green formula. The similar problems whosesolutionsare searched in the form of the sum of various potentials are investigated by O.G.Asadova in works [18],[19].

Let us assume

(i) The right hand side of boundary condition (8), $\widetilde{\alpha}\left(x_{1}, \lambda\right)$ is differentiable [of class $C^{(1)}\left(a_{1}, b_{1}\right)$ ] and vanishes at $a_{1}$ and $b_{1}$, i.e.

$$
\widetilde{\alpha}\left(a_{1}, \lambda\right)=\tilde{\alpha}\left(b_{1}, \lambda\right)=0
$$

(ii) $\widetilde{\alpha}_{1}\left(x_{1}\right), \widetilde{\alpha}_{2}\left(x_{1}\right)$ in boundary condition (8) are of Holder class.

(iii) $K_{1}\left(x_{1}, \xi_{1}\right), K_{2}\left(x_{1}, \xi_{1}\right)$ the kernels inside the integral term of (8) are continuous or they contain weak singularity.

Theorem 2. If assumptions (i), (ii), (iii) and conditions in Theorem 1 hold true then solution to boundary value problem (6) and (8) satisfies regularization relations (17).

Proof. Now we try to replace boundary $\Gamma$ with $\Gamma_{1}$ and $\Gamma_{2}$ introduced in section 1. Consider the last term of (13), we can write

$$
\begin{gathered}
\frac{1}{2} \tilde{u}\left(\xi_{1}, \gamma_{k}\left(\xi_{1}\right), \lambda\right)=\int_{D} F(x, \lambda) \bar{V}\left(x_{1}-\xi_{1}, x_{2}-\gamma_{k}\left(\xi_{1}\right), \lambda\right) d x+ \\
\int_{a_{1}}^{b_{1}} \tilde{u}\left(x_{1}, \gamma_{1}\left(x_{1}\right), \lambda\right) \bar{V}\left(x_{1}-\xi_{1}, \gamma_{1}\left(x_{1}\right)-\gamma_{k}\left(\xi_{1}\right), \lambda\right)\left[1-i \gamma_{1}^{\prime}\left(x_{1}\right)\right] d x_{1}- \\
\int_{a_{1}}^{b_{1}} \tilde{u}\left(x_{1}, \gamma_{2}\left(x_{1}\right), \lambda\right) \bar{V}\left(x_{1}-\xi_{1}, \gamma_{2}\left(x_{1}\right)-\gamma_{k}\left(\xi_{1}\right), \lambda\right)\left[1-i \gamma_{2}^{\prime}\left(x_{1}\right)\right] d x_{1} \\
k=1,2, \xi_{1} \in\left[a_{1}, b_{1}\right] .
\end{gathered}
$$

If the fundamental solution (11) is substituted into (15), in the resulting equality, then for $k=1$ the integral kernel of the second term and for $k=2$ the integral kernel of third term have singularity. Considering these terms we will find 


$$
\left\{\begin{array}{l}
\tilde{u}\left(\xi_{1}, \gamma_{1}\left(\xi_{1}\right), \lambda\right)=\frac{i}{\pi} \int_{a_{1}}^{b_{1}} \frac{\tilde{u}\left(x_{1}, \gamma_{1}\left(x_{1}\right), \lambda\right)}{x_{1}-\xi_{1}} d x_{1}+B_{1}\left(\xi_{1}, \lambda\right) \\
\widetilde{u}\left(\xi_{1}, \gamma_{2}\left(\xi_{1}\right), \lambda\right)=-\frac{i}{\pi} \int_{a_{1}}^{b_{1}} \frac{\tilde{u}\left(x_{1}, \gamma_{2}\left(x_{1}\right), \lambda\right)}{x_{1}-\xi_{1}} d x_{1}+B_{2}\left(\xi_{1}, \lambda\right)
\end{array}\right.
$$

where $B_{k}\left(\xi_{1}, \lambda\right), k=1,2$, are the functions with weak singular terms.

Remark 2: For those who are interested in numerical approach, one may write $B_{1}\left(\xi_{1}, \lambda\right)$ and $B_{2}\left(\xi_{1}, \lambda\right)$ in the following forms:

$$
\begin{gathered}
B_{1}\left(\xi_{1}, \lambda\right)=2 \int_{D} F(x, \lambda) \bar{V}\left(x_{1}-\xi_{1}, x_{2}-\gamma_{1}\left(\xi_{1}\right), \lambda\right)- \\
2 \int_{a_{1}}^{b_{1}} \tilde{u}\left(x_{1}, \gamma_{2}\left(x_{1}\right), \lambda\right) \bar{V}\left(x_{1}-\xi_{1}, \gamma_{2}\left(x_{1}\right)-\gamma_{1}\left(\xi_{1}\right), \lambda\right)\left(1-i \gamma_{2}^{\prime}\left(x_{1}\right)\right) d x_{1}- \\
\frac{1}{\pi} \int_{a_{1}}^{b_{1}} \frac{\gamma_{1}^{\prime}\left(\sigma_{1}\left(x_{1}, \xi_{1}\right)\right)-\gamma_{1}^{\prime}\left(x_{1}\right)}{1-i \gamma_{1}^{\prime}\left(\sigma_{1}\left(x_{1}, \xi_{1}\right)\right.} \frac{\tilde{u}\left(x_{1}, \gamma_{1}\left(x_{1}\right), \lambda\right)}{x_{1}-\xi_{1}} d x_{1}+ \\
\frac{i}{\pi} \int_{a_{1}}^{b_{1}} \frac{1-i \gamma_{1}^{\prime}\left(x_{1}\right)}{1-i \gamma_{1}^{\prime}\left(\sigma_{1}\left(x_{1}, \xi_{1}\right)\right)}\left\{e^{-i\left(x_{1}-\xi_{1}\right)\left[\tau \gamma_{1}^{\prime}\left(\sigma_{1}\left(x_{1}, \xi_{1}\right)-c\right]\right.}-1\right\} \tilde{u}\left(x_{1}, \gamma_{1}\left(x_{1}\right), \lambda\right) \frac{d x_{1}}{x_{1}-\xi_{1}}
\end{gathered}
$$

we also have

$$
\begin{gathered}
B_{2}\left(\xi_{1}, \lambda\right)=2 \int_{D} F(x, \lambda) \bar{V}\left(x_{1}-\xi_{1}, x_{2}-\gamma_{2}\left(\xi_{1}\right), \lambda\right)+ \\
2 \int_{a_{1}}^{b_{1}} \tilde{u}\left(x_{1}, \gamma_{1}\left(x_{1}\right), \lambda\right) \bar{V}\left(x_{1}-\xi_{1}, \gamma_{1}\left(x_{1}\right)-\gamma_{2}\left(\xi_{1}\right), \lambda\right)\left(1-i \gamma_{1}^{\prime}\left(x_{1}\right)\right) d x_{1}+ \\
\frac{1}{\pi} \int_{a_{1}}^{b_{1}} \frac{\gamma_{2}^{\prime}\left(\sigma_{2}\left(x_{1}, \xi_{1}\right)\right)-\gamma_{2}^{\prime}\left(x_{1}\right)}{1-i \gamma_{2}^{\prime}\left(\sigma_{2}\left(x_{1}, \xi_{1}\right)\right.} \frac{\tilde{u}\left(x_{1}, \gamma_{2}\left(x_{1}\right), \lambda\right)}{x_{1}-\xi_{1}} d x_{1}- \\
\frac{i}{\pi} \int_{a_{1}}^{b_{1}} \frac{1-i \gamma_{2}^{\prime}\left(x_{1}\right)}{1-i \gamma_{2}^{\prime}\left(\sigma_{2}\left(x_{1}, \xi_{1}\right)\right)}\left\{e^{-i\left(x_{1}-\xi_{1}\right)\left[\tau \gamma_{2}^{\prime}\left(\sigma_{2}\left(x_{1}, \xi_{1}\right)-c\right]\right.}-1\right\} \tilde{u}\left(x_{1}, \gamma_{2}\left(x_{1}\right), \lambda\right) \frac{d x_{1}}{x_{1}-\xi_{1}},
\end{gathered}
$$

where $\sigma_{1}\left(x_{1}, \xi_{1}\right), \sigma_{2}\left(x_{1}, \xi_{1}\right)$ are points between $x_{1}, \xi_{1}$.

Multiplying first and second terms of (16) by $\alpha_{1}\left(\xi_{1}\right)$ and $-\alpha_{2}\left(\xi_{1}\right)$ respectively and add two resulted equations. The right hand side of obtained equation have terms with strong singularity. Multipliers of these singularities (in the integral) are nonlocal terms in boundary condition (8). We determine these terms from (8) and by substitution we obtain.

$$
\begin{aligned}
& \alpha_{1}\left(\xi_{1}\right) \tilde{u}\left(\xi_{1}, \gamma_{1}\left(\xi_{1}\right), \lambda\right)-\alpha_{2}\left(\xi_{1}\right) \tilde{u}\left(\xi_{1}, \gamma_{2}\left(\xi_{1}\right), \lambda\right)= \\
& \frac{i}{\pi} \int_{a_{1}}^{b_{1}}\left\{\left[\alpha_{1}\left(\xi_{1}\right)-\alpha_{1}\left(x_{1}\right)\right] \tilde{u}\left(x_{1}, \gamma_{1}\left(x_{1}\right), \lambda\right)+\right. \\
& +\left[\alpha_{2}\left(\xi_{1}\right)-\alpha_{2}\left(x_{1}\right)\right] \tilde{u}\left(x_{1}, \gamma_{2}\left(x_{1}\right), \lambda\right\} \frac{d x_{1}}{x_{1}-\xi_{1}}+
\end{aligned}
$$




$$
\begin{gathered}
\alpha_{1}\left(\xi_{1}\right) B_{1}\left(\xi_{1}, \lambda\right)-\alpha_{2}\left(\xi_{1}\right) B_{2}\left(\xi_{1}, \lambda\right)+ \\
\frac{i}{\pi} \int_{a_{1}}^{b_{1}}\left[\alpha_{1}\left(x_{1}\right) \tilde{u}\left(x_{1}, \gamma_{1}\left(x_{1}\right), \lambda\right)+\alpha_{2}\left(x_{1}\right) \tilde{u}\left(x_{1}, \gamma_{2}\left(x_{1}\right), \lambda\right)\right] \frac{d x_{1}}{x_{1}-\xi_{1}}= \\
\frac{i}{\pi} \int_{a_{1}}^{b_{1}} \frac{d x_{1}}{x_{1}-\xi_{1}}\left\{\tilde{\alpha}\left(x_{1} \lambda\right)-\int_{a_{1}}^{b_{1}}\left[K_{1}\left(x_{1}, \eta_{1}\right) \tilde{u}\left(\eta_{1}, \gamma_{1}\left(\eta_{1}\right), \lambda\right)+K_{2}\left(x_{1}, \eta_{1}\right) \tilde{u}\left(\eta_{1}, \gamma_{2}\left(\eta_{1}\right), \lambda\right)\right] d \eta_{1}-\right. \\
\left.\int_{D} K\left(x_{1}, \eta\right) \tilde{u}(\eta, \lambda) d \eta\right\}+\alpha_{1}\left(\xi_{1}\right) B_{1}\left(\xi_{1}, \lambda\right)-\alpha_{2}\left(\xi_{1}\right) B_{2}\left(\xi_{1}, \lambda\right)+ \\
\frac{i}{\pi} \int_{a_{1}}^{b_{1}}\left\{\left(\alpha_{1}\left(\xi_{1}\right)-\alpha_{1}\left(x_{1}\right)\right) \tilde{u}\left(x_{1}, \gamma_{1}\left(x_{1}\right), \lambda\right)+\left[\alpha_{2}\left(\xi_{1}\right)-\alpha_{2}\left(x_{1}\right)\right] \tilde{u}\left(x_{1}, \gamma_{2}\left(x_{1}\right), \lambda\right)\right\} \frac{d x_{1}}{x_{1}-\xi_{1}}
\end{gathered}
$$

Remark 3: It is obvious that assumptions (i)-(iii) are sufficientto keep right hand side of (17) regular. The first term of right hand side in (17) needs (i), second and third term need (iii) and the last term needs (ii) to be held.

\section{Fredholm's Type}

Regularization relation (17) plus boundary condition (8) make a system of integral equations with unknowns $\tilde{u}\left(x_{1}, \gamma_{k}\left(x_{1}\right), \lambda\right), k=1,2$. If conditions (i)-(iii) and the following condition are satisfied:

(iv). in boundary condition (8) the multipliers $\alpha_{k}\left(x_{1}\right) \neq 0, k=1,2$, where $x_{1} \in\left[a_{1}, b_{1}\right]$ then the above system of integral equations can be transformed to the normal (canonical) form, where kernels of these integrals contain weak singularity. Hence the following theorem holds:

Theorem 3: If additional assumption (iv) to conditions in theorem 2 holds, then the boundary value problem (6) and (8) is of Fredholm's type.

Proof: Indeed system (8) and (17) with respect to function $\tilde{u}\left(x_{1}, \gamma_{k}\left(x_{1}\right), \lambda\right), k=1,2$ is a linear system and is linear dependent to function $\tilde{u}(x, \lambda), x \in D$. If this system of equations has unique solution then it is in the form $\tilde{u}\left(x_{1}, \gamma_{k}\left(x_{1}\right), \lambda\right)$ and it is linearly dependent to function $\tilde{u}(x, \lambda), x \in D$. Now consider the solution obtained from the above discussion and substitute it into the first relation of $(13)$, hence $\tilde{u}(x, \lambda)$, which is a Fredholm integral equation of second type, is obtained. This is a proof that boundary condition problem (6) and (8) is of Fredholm's type.

Remark 4: Aliev and Ebadpour [1] considered the boundary value problem (6) and (8) for the special case $\lambda=0$. They proved that for $\lambda=0$ problem (6) and (8) is of Fredholm's type.

\subsection{Solution to Problem and Its Asymptotic}

Theorem 4: Let conditions in the theorem 3 holds, if $a(x)$ is continuous, $\varphi(x) \equiv 0$ and functions $f(x, t)$,

$\alpha\left(x_{1}, t\right)$ satisfy in condition (v) then for large enough absolute values of $\lambda$, solution of problem (6) and (8) exists and for this solution, the following asymptotic will obtain:

$$
|\widetilde{u}(x, \lambda)|=O\left(|\lambda|^{-3}\right), x \in D
$$

Proof: We can write $(x, t)$ is three times differentiable with respect to $t$. If we also assume that
(v). Function

$$
f(x, 0)=\left.\frac{\partial f(x, t)}{\partial t}\right|_{t=0}=0,
$$

In section 3, we explained how the solution of boundary value problem (6) and (8) will be obtained from the first relation of (13), i.e. 


$$
\begin{gathered}
\tilde{u}(\xi, \lambda)=-\int_{D} a(x) \bar{V}(x-\xi, \lambda) \tilde{u}(x, \lambda) d x-\int_{D} \phi(x) \bar{V}(x-\xi, \lambda) d x- \\
\int_{D} \tilde{f}(x, \lambda) \bar{V}(x-\xi, \lambda) d x+\int_{a_{1}}^{b_{1}} \tilde{u}\left(x_{1}, \gamma_{1}\left(x_{1}\right), \lambda\right) \bar{V}\left(x_{1}-\xi_{1}, \gamma_{1}\left(x_{1}\right)-\xi_{2}, \lambda\right)\left(1-i \gamma_{1}^{\prime}\left(x_{1}\right)\right) d x_{1}- \\
\int_{a_{1}}^{b_{1}} \tilde{u}\left(x_{1}, \gamma_{2}\left(x_{1}\right), \lambda\right) \bar{V}\left(x_{1}-\xi_{1}, \gamma_{2}\left(x_{1}\right)-\xi_{2}, \lambda\right)\left(1-i \gamma_{2}^{\prime}\left(x_{1}\right)\right) d x_{1} \quad \xi \in D
\end{gathered}
$$

The third term (20) for large enough $|\lambda|$ satisfies the relationship

$$
\left|\int_{D} \widetilde{f}(x, \lambda) \bar{V}(x-\xi, \lambda) d x=O\left(|\lambda|^{-3}\right)\right| .
$$

Substituting fundamental solution (11) in (20), we obtain asymptotic of problem (6) and (8). Without losing generality, we assume

$$
\varphi(x)=0, x \in D
$$

this forces problem (1) and (2) not to be homogeneous. This implies that the second term in (20) vanishes.

To get asymptotic from third term of (20), we first write (5) in the following way:

$$
\begin{gathered}
\widetilde{f}(x, \lambda)=\int_{0}^{\infty} e^{-\lambda t} f(x, t) d t=\left.f(x, t) \frac{e^{-\lambda t}}{-\lambda}\right|_{t=0} ^{\infty}+ \\
\frac{1}{\lambda} \int_{0}^{\infty} e^{-\lambda t} \frac{\partial f(x, t)}{\partial t} d t=\frac{f(x, 0)}{\lambda}+\frac{1}{\lambda}\left[\left.\frac{\partial f(x, t)}{\partial t} \frac{e^{-\lambda t}}{-\lambda}\right|_{t=0} ^{\infty}+\right. \\
\left.\frac{1}{\lambda} \int_{0}^{\infty} e^{-\lambda t} \frac{\partial^{2} f(x, t)}{\partial t^{2}} d t\right]=\frac{f(x, 0)}{\lambda}+\left.\frac{1}{\lambda} \frac{\partial f(x, t)}{\partial t}\right|_{t=0}+ \\
\frac{1}{\lambda^{2}}\left[\left.\frac{\partial^{2} f(x, t)}{\partial t^{2}} \frac{e^{-\lambda t}}{-\lambda}\right|_{t=0} ^{\infty}+\frac{1}{\lambda} \int_{0}^{\infty} e^{-\lambda t} \frac{\partial^{3} f(x, t)}{\partial t^{3}} d t\right]
\end{gathered}
$$

If $|\tilde{u}(x, \lambda)|$ is bounded for the largest absolute value of $\lambda$ then the first term in R.H.S. of (20) converges to zero with the speed of Fourier multipliers. Similarly, the last two terms in R.H.S. of (20) converges to zero with the same speed. If $\left|\widetilde{u}\left(x_{1}, \gamma_{k}\left(x_{1}\right), \lambda\right)\right|$ for $k=1,2$ is bounded then from (20) we obtain:

$$
\lim _{\tau \rightarrow+\infty} \tilde{u}(x, \lambda)=0, x \in D .
$$

Now if we ignore all those terms with singularity in (15), we may write

$$
\lim _{\tau \rightarrow+\infty} \tilde{u}\left(x_{1}, \gamma_{k}\left(x_{1}\right), \lambda\right)=0, x_{1} \in\left[a_{1}, b_{1}\right], k=1,2
$$

Remark 5: Existence and uniqueness of the solution to Schrodinger equation (an equation which is independent of time and dependent to parameter) with nonlocal boundary condition is proved by Kavei and Aliev [13].

\subsection{Solution to Mixed Problem}

With the help of Laplace transformation the solution to mixed problem (1-3) may be written as follows ([4], [11]): 


$$
u(x, t)=\frac{1}{2 \pi i} \int_{c-i \infty}^{c+i \infty} e^{\lambda t} \tilde{u}(x, \lambda) d \lambda
$$

We considered in (18) that the solution of (24) and first derivatives (with respect to $x_{1}, x_{2}$ and $t$ ) exist, i.e. in (24) we may let derivative appear inside integral.

Theorem 5: Let conditions in theorem 4 hold true, the solution to complex problem (1-3) exists, is unique and is in the form (24).

Proof: Substituting (24) into equation (1) gives ([12]):

$$
\begin{aligned}
\frac{\partial u}{\partial t}- & \frac{\partial u}{\partial x_{2}}-i \frac{\partial u}{\partial x_{1}}=\frac{1}{2 \pi i} \int_{c-i \infty}^{c+i \infty} e^{-\lambda t}\left\{\lambda \tilde{u}-\frac{\partial \tilde{u}}{\partial x_{2}}-i \frac{\partial u}{\partial x_{1}}\right\} d \lambda= \\
& -\frac{1}{2 \pi i} \int_{c-i \infty}^{c+i \infty} e^{-\lambda t} F(x, \lambda) d \lambda=\frac{1}{2 \pi i} \int_{c-i \infty}^{c+i \infty} e^{-\lambda t} \tilde{f}(x, \lambda) d \lambda+ \\
& \frac{1}{2 \pi i} \int_{c-i \infty}^{c+i \infty} e^{\lambda t} a(x) \tilde{u}(x, \lambda) d \lambda=f(x, t)+a(x) u(x, t) .
\end{aligned}
$$

This means that relation (24) satisfies equation (1).

Now substitute (24) into the left hand side of (2), then we obtain:

$$
\begin{gathered}
\alpha_{1}\left(x_{1}\right) u\left(x_{1}, \gamma_{1}\left(x_{1}\right), t\right)+\alpha_{2}\left(x_{1}\right) u\left(x_{1}, \gamma_{2}\left(x_{1}\right), t\right)+ \\
\int_{a_{1}}^{b_{1}}\left[K_{1}\left(x_{1}, \xi_{1}\right) u\left(\xi_{1}, \gamma_{1}\left(\xi_{1}\right), t\right)+K_{2}\left(x_{1}, \xi_{1}\right) u\left(\xi_{1}, \gamma_{2}\left(\xi_{1}\right), t\right)\right] d \xi_{1}+ \\
\int_{D} K\left(x_{1}, \xi\right) u(\xi, t) d \xi=\frac{1}{2 \pi i} \int_{c-i \infty}^{c+i \infty} e^{\lambda t}\left\{\alpha_{1}\left(x_{1}\right) \tilde{u}\left(x_{1}, \gamma_{1}\left(x_{1}\right), \lambda\right)+\right. \\
\alpha_{2}\left(x_{1}\right) \tilde{u}\left(x_{1}, \gamma_{2}\left(x_{1}\right), \lambda\right)+\int_{a_{1}}^{b_{1}}\left[K_{1}\left(x_{1}, \xi_{1}\right) u\left(\xi_{1}, \gamma_{1}\left(\xi_{1}\right), \lambda\right)+K_{2}\left(x_{1}, \xi_{1}\right) u\left(\xi_{1}, \gamma_{2}\left(\xi_{1}\right), \lambda\right)\right] d \xi_{1}+ \\
\left.\int_{D} K\left(x_{1}, \xi\right) u(\xi, \lambda) d \xi\right\} d \lambda=\frac{1}{2 \pi i} \int_{c-i \infty}^{c+i \infty} e^{\lambda t} \tilde{\alpha}\left(x_{1}, \lambda\right) d \lambda=\alpha\left(x_{1}, t\right)
\end{gathered}
$$

i.e. relation (24) satisfies boundary condition (2) ([12]).

Finally by substituting relation (24) into (3) we obtain

$$
u(x, 0)=\frac{1}{2 \pi i} \int_{c-i \infty}^{c+i \infty} \tilde{u}(x, \lambda) d \lambda .
$$

From fundamental solution (11) and asymptotic relation (18) we observe that if we change the Laplace line with the limits of right half side circles, with the help of Jordan Lemma ([14], [15]) it is obvious that relation (25) vanishes. Therefore, we may write down the following theorem for the mixed and complex problem (1-3).

\section{Related Open Problems}

(a) Verifying boundary value problem (6) and (8) where boundary $\Gamma$ of region $D$ is not Lyapunov curve.

$$
\left(\gamma_{k}\left(x_{1}\right) \in C^{(1)}\left(\left[a_{1}, b_{1}\right]\right), \quad k=1,2\right)
$$

(b) Verifying problem (6) and (8) where $\Gamma$ is Lyapunov but $D$ is not a connected region. 
(c) Finding the solution for the following problem:

$$
\frac{\partial u(x)}{\partial x_{3}}=\frac{\partial u(x)}{\partial x_{2}}+i \frac{\partial u(x)}{\partial x_{1}}, x=\left(x_{1}, x_{2}, x_{3}\right) \in D \subset I R^{3}
$$

with boundary condition

$$
\begin{gathered}
\sum_{j=1}^{2}\left[\alpha_{j}\left(x^{\prime}\right) u\left(x^{\prime}, \gamma_{j}(x)\right)+\int_{S} K_{j}\left(x^{\prime}, \xi^{\prime}\right) u\left(\xi^{\prime}, \gamma_{j}\left(\xi^{\prime}\right)\right) d \xi^{\prime}\right]+ \\
\int_{D} K\left(x^{\prime}, \xi\right) u(\xi) d \xi=\alpha\left(x^{\prime}\right)
\end{gathered}
$$

where $x^{\prime}=\left(x_{1}, x_{2}\right) \in S \subset I R^{2}$ and $\Gamma_{k}: x_{3}=\gamma_{k}\left(x^{\prime}\right), k=1,2, x^{\prime} \in S, i=\sqrt{-1}$

\section{REFERENCES}

[1] Aliev N. and Ebadpour J., Investigation to Fredhols type initial boundary value problem containing elliptic D.E. of first order with nonlocal and global boundary conditions, 31 Iranian Mathematical Annual, Tehran University, 1998 (In Persian).

[2] Aliev N., Some open problems in theory of initial value problems for mathematics and physics, 26 Iranian Mathematical Annual, Tabriz University, 19958 (In Persian).

[3] Vladimirovi V.S., Equations of Mathematical Physics, Mir Publishers Moscow, 1984.

[4] Courant R. and Hilbert D., Methods of Mathematical Physici, New York, London, 1962.

[5] Petrovski I.G., Lectures on Partial Differential Equations, Interscience, New York, 1954.

[6] Tikhonov A.N., Samarski A.A., Equations of Mathematical Physics, Dover Publications, Inc. New York, 1990.

[7] Aliev N., and Jahanshahi M., Sufficient conditions for Reduction of The BVP including a Mixed P.D.E with nonlinear boundary conditions to Fredholm Integral Equation, JNT.J.Math.Educ. Tech. Scie. vol.28, No.3, 1997.

[8] Kavei G., and Aliev N., An analytic method to the solution time dependent Schrodinger equation using half cylinder space system-I, Bulletin of Pure and Applied Sciences, vol.1.16, No.2, 1997.

[9] Deniz A.A., Partial differential equations, Spreinger-Varlag. New York, 1987.

[10] Hormander L., On the theory of general partial differential operators, Acta. Mathematica, 4(1955), 161-248.

[11] Tranter C.J., Integral transforms on mathematical physics, John Wiley and Suns; New York, 1951

[12] Rasulov M.L., Methods of contour integration, North-Holland Publishing Company-Amsterdam, 1967.

[13] Kavei G., and Aliev N., The existence and uniqueness of the solution of the spectral problem II, J.Sci. I.R.Iran. vol.1.10, No.4, Autumn 1999.

[14] Evgrafov M.A., Analitic functions, Dover represent 1978, (translated from the Russian).

[15] Lavrent'ev M.A., and Shabat B.V., Methods for complex functions theories, Dentsch, VarligWissenschoff, 1968, (translated from the Russian).

[16] Kostyuchenko A.G., Krasnosel'skii M.A., Kreyn S.G., Maslov V.P., Sobolov V.I., and Faddeev L.D., Functional analysis, Nauka, Moscow, 1964, (translated from the Russian).

[17] Aliev N.,Hosseini S.M., An analysis of a parabolic problem with a general (Non-local and global) supplementary linear conditions, I, Italian Journal of Pure and Applied Mathematics, No.12-2002, 143-154.

[18] Asadova O.H. Mark of decisions of spectral problems, corresponding to problems for the parabolic equation of an order more than two, Differential equations, p. 1839, Minsk 1974.

[19] Asadova O.H. On one boundary problem for the 6-th order polyharmonic equations. Proceedings of IMM of NAS of Azerbaijan, page 22, 2001. 